\title{
Developmental quotient at 24 months and fatty acid composition of diet in early infancy: a follow up study
}

Carlo Agostoni, Sabina Trojan, Roberto Bellù, Enrica Riva, Maria Grazia Bruzzese, Marcello Giovannini

\begin{abstract}
Aim-A follow up study of developmental quotient (DQ) at 24 months of toddlers whose diets in early infancy differed in fatty acid composition, and in whom an association between diet and DQ was observed at 4 months.

Methods-81 toddlers were distributed among three groups according to early type of diet: standard infant formula (SFo, $\mathbf{n}=30$ ); long chain polyunsaturated fatty acid (LC-PUFA) enriched formula (LCPFo, $n=26)$; human milk (HM, $n=$ 25). DQ at 24 months was assessed by Brunet-Lézine's psychomotor developmental test. A subgroup ( $n=20$; SFo 8; LCPFo 6; HM 6) was tested for erythrocyte phosphatidylcholine and phosphatidylethanolamine.
\end{abstract}

Results-No DQ differences were found by analysis of variance. Neither DQ nor erythrocyte docosahexaenoic acid at 4 months were predictors of DQ scores at 24 months. Phosphatidylcholine arachidonic and docosahexaenoic acid correlated positively, and phosphatidylcholine linoleic acid and phosphatidylethanolamine eicosapentaenoic acid negatively, with DQ. Multiple regression analysis including these variables explained $52 \%$ of interindividual DQ variance. A strong association was found between the erythrocyte phosphatidylcholine arachidonic/ linoleic acid ratio and DQ $(r=0.75 ; p=$ 0.0001).

Conclusions-The diet/DQ association found at 4 months was not predictive of DQ scores at $\mathbf{2 4}$ months. Irrespective of dietary or genetic factors, there appears to be a strong correlation between the LC-PUFA composition of the red cell membrane and higher neurodevelopmental performance.

(Arch Dis Child 1997;76:421-424)

Keywords: developmental quotient; toddlers; long chain polyunsaturated fatty acids; arachidonic acid

Arachidonic acid (C20:4 n-6), eicosapentaenoic acid (C20:5 n-3), docosahexaenoic acid (C22:6n-3), together with the essential linoleic (C18:2n-6) and $\alpha$ linolenic acids (C18:3n-3) are the most relevant polyunsaturated fatty acids (PUFA) in feeding for growth. ${ }^{1}$ Assuming that C20:4 n-6 and C22:6 n-3 are preferen- tially accumulated in the cerebral cortex phospholipids during lactation ${ }^{2}$ and that breast feeding is to be preferred in developmental terms, ${ }^{3}{ }^{4}$ we investigated the developmental effect of PUFA in a formula trial. ${ }^{5}$ In 1995 we reported a scoring advantage on psychomotor testing in a population of full term, 4 month old infants whose early diets had differed in fatty acid composition and whose infant formula had included C20:4 n-6 and C22:6 n-3. ${ }^{5}$ A direct relation between erythrocyte phospholipid C22:6 n-3 and developmental quotient (DQ)was later reported. ${ }^{6}$ Clinical studies of term infants' visual evoked potentials at 16 and 30 weeks $^{7}$ and of performance on neurodevelopmental test of 12 month olds born preterm ${ }^{8}$ were consistent with this latter observation.The population we had studied was reassessed at a 24 month follow up for the association between DQ and (1) the type of early diet and (2) the erythrocyte fatty acid pattern (in a subgroup of 20), to investigate the outcome of this short term association.

\section{Methods}

Ninety healthy infants of both sexes born between September 1992 and August 1993 were recruited in an ethically approved, randomised, parallel group, prospective study, the design of which has been described elsewhere. $^{5}$

Sixty mothers did not breast feed: their infants were randomly assigned to a group fed either Aptamil (a standard infant formula; group SFo, $n=31$ ), or Aptamil with Milupan, a fat modifying supplement including long chain (LC) PUFA (group LCPFo, $n=29$ ). Both formulas were supplied by Milupa of Friedrichsdorf, Germany. As the reference group, 30 mothers exclusively breast fed their infants for at least four months (group HM).

Gestational age, weight, length, and Italian census socioeconomic indicators (father's occupation and mother's education) were recorded at birth. Growth and DQ were monitored at 4,12, 18, and 24 months. Parents gave their informed consent and were counselled about the introduction of solid foods. A weaning formula was used from the fifth month by all the bottle fed infants. In the HM group breast feeding was encouraged throughout the weaning period and a weaning formula was introduced when lactation began to fail. Whole cows' milk was introduced during the 12th month. 
Table 1 DQ score (mean(SD)) at 24 months and infant dietary groups

\begin{tabular}{|c|c|c|c|c|c|}
\hline \multicolumn{4}{|c|}{ LCPFo (26) } & \multicolumn{2}{|c|}{ SFo (30) } \\
\hline \multicolumn{2}{|l|}{ DQ, mean (SD), 95\% CI } & \multicolumn{2}{|c|}{100.1 (10.3), 96.1 to 104.0} & \multicolumn{2}{|c|}{$99.1(7.1), 95.0$ to 103} \\
\hline \multicolumn{6}{|c|}{$\begin{array}{l}\text { LCPFo }=\text { infants fed with formula enriched with long chain polyunsaturated fatty acids; } S F o=\text { inf } \\
\text { CI = confidence interval. }\end{array}$} \\
\hline \multicolumn{6}{|c|}{$\begin{array}{l}\text { Table } 2 \text { Index of agreement of } D Q \text { at } 24 \text { months and } D Q \text { at } 4 \text { months according to } \\
\text { tertiles (number of subjects, } \% \text { in each tertile) }\end{array}$} \\
\hline & \multirow[b]{2}{*}{ Tertiles } & \multicolumn{4}{|c|}{ DQ at 24 months } \\
\hline & & 1 & 2 & 3 & Row total \\
\hline \multicolumn{6}{|l|}{ DQ at 4 months } \\
\hline De at t morths & \multirow[t]{2}{*}{1} & 9 & 10 & 8 & 27 \\
\hline & & $33.3 \%$ & $37 \%$ & $29.6 \%$ & $33.3 \%$ \\
\hline & \multirow[t]{2}{*}{2} & & 9 & 10 & \\
\hline & & $29.6 \%$ & $33.3 \%$ & $37.0 \%$ & $33.3 \%$ \\
\hline & \multirow[t]{2}{*}{3} & 10 & & & \\
\hline & & $37 \%$ & $29.6 \%$ & $33.3 \%$ & $33.3 \%$ \\
\hline & \multirow[t]{2}{*}{ Column total } & & & 27 & 81 \\
\hline & & $33.3 \%$ & $33.3 \%$ & $33.3 \%$ & $100 \%$ \\
\hline
\end{tabular}

$\kappa=0.000, \mathrm{p}=\mathrm{NS}$.

At 24 months Brunet-Lézine's scale (adjusted and standardised for 1 to 30 month olds) ${ }^{9}$ was used to calculate DQ. Whole venous blood samples were obtained from 20 infants after obtaining informed parental consent. Different profiles of the erythrocyte phospholipid LC-PUFA pool have been associated with cerebral cortex tissue composition ${ }^{10}$ and function ${ }^{6}$ and thus membrane phosphatidylcholine and phosphatidylethanolamine were factored into the 24 month analysis.

CHEMICAL ANALYSES

After sample preparation, ${ }^{5}$ erythrocyte phospholipid fractions were separated on silica gel plates (Merck, Darmstadt, Germany) in chloroform $/ \mathrm{methanol} /$ glacial acetic acid/water (50:30:10:3 vol/vol/vol/vol). Egg yolk phosphatidylcholine and bovine brain phosphatidylethanolamine (from Sigma Aldrich, Stanheim, Germany) were used as standards. Fatty acids were transesterified with methanolic hydrochloride and analysed by high resolution gas chromatography (Mega 2 GC, Fisons Instruments, Rodano, Italy), equipped with a flame ionisation detector and a fused silica capillary column (Supelco Omegawax 320, 30 $\mathrm{m}$ in length, $0.32 \mathrm{~mm}$ internal diameter, and $0.25 \mu \mathrm{m}$ thick film). Column temperature programming was increased from $60^{\circ} \mathrm{C}$ to $150^{\circ} \mathrm{C}$ by $10^{\circ} \mathrm{C} / \mathrm{min}$ increments, from $150^{\circ} \mathrm{C}$ to $170^{\circ} \mathrm{C}$ by $5^{\circ} \mathrm{C} / \mathrm{min}$ increments, and from $170^{\circ} \mathrm{C}$ to $235^{\circ} \mathrm{C}$ by $2^{\circ} \mathrm{C} / \mathrm{min}$ increments and held for 30 minutes. Individual detector outputs were identified using pure reference compounds and by mass spectrometry (Trio 1000 , Fisons Instruments, Italy) and expressed as weight percentage of fatty acid methyl esters.

Peak areas were calculated on Chrom-Card software using heptadecanoic acid as internal standard. The detected molecules were C16:0, C18:0, and C24:0 in the saturated class, C16:1 $\mathrm{n}-7, \mathrm{C} 18: 1 \mathrm{n}-9, \mathrm{C} 18: 1 \mathrm{n}-7$, and C24:1 n-9 in the monounsaturated, and C18:2 n-6, C18:3 n-6, C18:3 n-3, C20:3 n-9, C20:3 n-6, C20:4 n-6, C20:5 n-3, C22:4 n-6, C22:5 n-6, C22:5 $n-3$, and C22:6n-3 among the polyunsaturated groups.
STATISTICAL ANALYSIS

In designing the study a $10 \%$ between group variation in DQ score was allowed to define a clinically acceptable difference with the probability $\beta$ of a type II error fixed at 0.10 , and sample size for that magnitude was calculated at 24 per group. For the purpose of comparison, the data were expressed as the group means (SD) of DQ values. Between group differences in DQ were tested by analysis of variance. The Kruskal-Wallis procedure was used to test between group fatty acid differences. The $\kappa$ statistic was used to determine the degree of agreement between DQ scores at 4 and 24 months. Categorical variables were compared by the $\chi^{2}$ and Fisher's exact test procedures. Correlations between fatty acid variables and DQ scores were tested by Pearson correlation coefficients. The multiple regression equation was derived by modelling DQ as response (dependent) variable and the fatty acid linked to DQ (with $\mathrm{p}<0.10$ ) by bivariate analysis as explanatory (independent) variables. All analyses were made with the SPSS/PC+ 3.1 statistical computer package (SPSS Inc, Chicago, IL, USA) except the regression analysis with the confidence ellipse, which was performed with SAS (SAS Institute, Cary, NC, USA).

\section{Results}

Five infants out of a total of 86 tested at 4 months were lost to follow up 20 months later because of relocation ( $n=2)$ or recurrent infection $(n=3)$. The final sample included 81 subjects: 30 in the SFo group, 26 in the LCPFo group, and 25 in the HM group. The sample mean score on the Brunet-Lézine test was 99.6 (SD 8.2), 95\% confidence interval $(\mathrm{CI})=97.8$ to 101.4 , range $=83.8$ to 122.1 . Percentile cutoffs were set at the following points: $3 \%, 87.4$; $10 \%, 90.3 ; 25 \%, 92.4 ; 50 \%, 98.7 ; 75 \%, 105.3$; $90 \%, 111.2 ; 97 \%, 118.7$.

DQ performance did not differ among the dietary groups (table 1). The tertile distribution of the DQ value at 4 months failed to predict tertiles of DQ value at 2 years of age (table 2). Erythrocyte DHA levels at 4 months, which

Table 3 Coefficients of correlation between major polyunsaturated fatty acids in erythrocyte phosphatidylcholine (PC) and phosphatidylethanolamine $(P E)$ and $D Q$ in 20 infants at 24 months

\begin{tabular}{lrl}
\hline Fatty acid & $r$ & $p$ \\
\hline Erythrocyte PC & -0.39 & 0.08 \\
C18:2 n-6 & 0.62 & 0.003 \\
C20:4 n-6 & 0.05 & 0.82 \\
C20:5 n-3 & 0.46 & 0.03 \\
C22:6 n-3 & & \\
Erythrocyte PE & -0.04 & 0.85 \\
C18:2 n-6 & -0.08 & 0.72 \\
C20:4 n-6 & -0.51 & 0.02 \\
C20:5 n-3 & 0.09 & 0.68 \\
C22:6 n-3 & & \\
\hline
\end{tabular}


Table 4 Multiple regression model including polyunsaturated fatty acids related to $D Q$ $(p<0.10)$ : variables in the equation

\begin{tabular}{llll}
\hline Variable & $B$ & $\beta$ & $p$ \\
\hline C20:4 n-6 in erythrocyte PC & 2.08 & 0.60 & 0.002 \\
C18:2 n-6 in erythrocyte PC & -1.10 & -0.36 & 0.04 \\
\hline
\end{tabular}

Multiple $R=0.721 ; R^{2}=0.520 ; \mathrm{F}=9.23 ; \mathrm{p}=0.001$.

$\mathrm{PC}=$ phosphatidylcholine.

we had found to correlate with $\mathrm{DQ}$ scores at 4 months, ${ }^{6}$ did not correlate with DQ when tested at 24 months.

The 20 blood sample donors ( $\mathrm{SFo}, \mathrm{n}=8$; LCPFo, $n=6 ; H M, n=6$ ) were comparable to other subjects for gestational age, gender distribution, weight, length, socioeconomic factors at birth and 24 months, and for DQ at 24 months. The subgroup fatty acid levels did not differ for major PUFA and fatty acid classes of erythrocyte phosphatidylcholine and phosphatidylethanolamine (data not shown). Correlation coefficients $(r)$ between major PUFA classes and DQ scores are given in table 3. The multiple regression equation into which variables related to $\mathrm{DQ}(\mathrm{p}<0.10)$ were modelled accounted for $52 \%$ of the interindividual DQ score variance (table 4). Erythrocyte phosphatidylcholine C20:4 n-6 was found to correlate positively, and $\mathrm{C} 18: 2 \mathrm{n}-6$ negatively, to DQ score. No n-3 series LC-PUFA significantly correlated with DQ in this model. A regression analysis modelling the erythrocyte phosphatidylcholine C20:4 n-6/C18:2 n-6 ratio and DQ yielded an $r$ value of 0.75 ( $\mathrm{p}=$ 0.0001 ) (fig 1).

Some caution should be used in the interpretation of this relation as we cannot rule out the possibility that the five children with a C20:4 n-6/C18:2 n-6 ratio in favour of the derived moiety form a cluster. However, this group of children was not from the same original dietary group and could not be differentiated from the other 15 on anthropometric characteristics or socioeconomic factors; therefore we consider the relation to be a true one.

\section{Discussion}

The relation between LC-PUFA status and neurodevolopmental performance has not previously been surveyed in a population of toddlers born at term. In this follow up study,

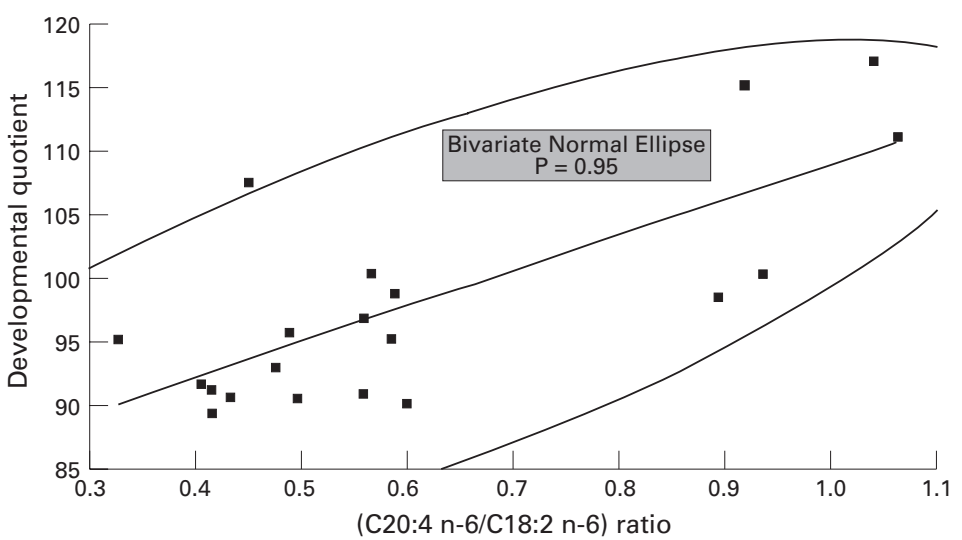

Figure 1 Erythrocyte phosphatidylcholine (C20:4 n-6/C18:2 n-6) ratio and developmental quotient: regression analysis and confidence ellipse. we report that the performance advantage scored by a sample of 4 month olds whose early diet had included LC-PUFA from human milk or a formula does not translate into a similar advantage at 24 months. The interpretation of this finding as the absence of a measurable outcome in the medium term remains an open question, but it would be consistent with the sample size required to obtain clinically significant between group variations of $\geqslant 10 \%$ in DQ scores at 4 months. ${ }^{5}$ Similarly, the fact that DQ at 4 months cannot be used reliably as a predictor of performance at reassessment agrees with the published reports on neurodevelopmental measurements. ${ }^{11}{ }^{12}$ The multivariate regression model used shows that with our data unsaturated fatty acid composition of erythrocyte phosphatidylcholine at 24 months correlates with interindividual variability of DQ scores. The composition of the erythrocyte membrane fatty acid pool could reflect both dietary and genetic influences.

Concomitantly, a strong positive correlation between the C20:4 n-6/C18:2 n-6 ratio in erythrocyte phosphatidylcholine and the DQ scores was found in this follow up analysis. Necropsies have shown similarities between erythrocyte lipid and brain cell fatty acid composition. ${ }^{10}$ An increase in membrane phosphatidylcholine and phosphatidylethanolamine unsaturation modulates the activity of membrane bound enzymes, ${ }^{13}$ and changes in phospholipid acyl chain unsaturation could be involved with cell metabolism and the physiological control of receptor response. The disappearance of the association between C22:6 n-3 and DQ from the multivariate equation should not detract from the neurobiological role of C22:6 n-3. Its synthesis from the substrate C18:3 n-3 shares enzymatic steps ( $\Delta-6$ and $\Delta-5$ desaturases) with the synthesis of C20:4 n-6 from C18:2 n-6, and therefore levels of synthesised C22:6 n-3 and C20:4 n-6 could be mutually dependent. ${ }^{14} 15$

Our data support the interpretation that the early diet/DQ correlation does not take on medium term predictive significance. However, at 24 months the association between the current accretion of LC-PUFA in erythrocyte membrane phosphatidylcholine and the toddler's individual response to external stimuli persists. Studies taking account of current dietary intakes are needed to interpret the relation between the fatty acid composition of membranes and neurodevelopmental performance.

We thank Gabriel R Bouygue MA for his critical assistance during the preparation of the manuscript.

1 Giovannini M, Riva E, Agostoni C. Fatty acids in paediatric nutrition. Pediatr Clin North Am 1995;42:861-77.

2 Farquharson J, Cockburn F, Patrick WA, Jamieson EC, Logan RW. Infant cerebral cortex phospholipid fatty-acid composition and diet. Lancet 1992;340:810-3.

3 Lucas A, Morley R, Cole TJ, Lister G, Leeson Pajne C. Breast milk and subsequent intelligence quotient in children born preterm. Lancet 1992;339:261-4.

4 Pollock JI. Long-term associations with infant feeding in a clinically advantaged population of babies. Dev Med Child

5 Agostoni C, Trojan S, Bellù R, Riva E, Giovannini M. Neurodevelopmental quotient of healthy term infants and feed- 
ing practice: the role of long-chain polyunsaturated fatty acids. Pediatr Res 1995;38:262-6.

6 Agostoni C, Riva E, Trojan S, Bellù R, Giovannini M. Docosahexaenoic acid status and developmental quotient of healthy term infants. Lancet 1995;346:868

7 Makrides M, Neumann M, Simmer K, Pater J, Gibson R. Are long-chain polyunsaturated fatty acids essential nutrients in infancy? Lancet 1995;345:1463-8.

8 Bjerve KS, Brubakk AM, Fougner KJ, Johnsen H, Midthjell $\mathrm{K}$, Vit T. Omega-3 fatty acids: essential fatty acids with important biological effects, and serum phospholipid fatty acids as markers of dietary $\omega-3$ fatty acid intake. Am $\mathcal{F}$ Clin Nutr 1993;57:801-6S.

9 Brunet O, Lézine I. Le développement psychologique de la première enfance. 2nd Ed. Paris: Presses Universitaires de France, 1966 (Italian translation, 1967).

10 Makrides M, Neumann MA, Byard RW, Simmer K, Gibson RA. Fatty acid composition of brain, retina, and erythro- cytes in breast- and formula-fed infants. Am $\mathcal{f}$ Clin Nutr 1994;60:189-94.

11 Amiel Tison C, Grenier A. Evaluation neurologique du nouveau-né et du nourisson. Paris: Masson, 1980.

12 Slater A. Individual differences in infancy and later IQ. $f$ Child Psychol Psychiatry 1995;36:69-112.

13 Orlacchio A, Maffei C, Binaglia L, Porcellati G. The effect of membrane acyl-chain composition on the activity of brain $\beta$-N-acetyl-D-glucosaminidase. Biochem 7 1981;195: 383-8.

14 Holman RT. Nutritional and metabolic interrelationships between fatty acids. Fed Proc 1964;23:1062-7.

15 Voss A, Reinhart M, Sankarappa S, Sprecher H. The metabolism of $7,10,13,16,19$-docosapentaenoic acid to $4,7,10,13,16,19$-docosahexaenoic acid in rat liver is independent of a 4-desaturase. F Biol Chem 1991;266: 19995-20000. 